\title{
Dielectric and photo-dielectric properties of TIGaSeS crystals
}

\author{
A F QASRAWI ${ }^{\mathrm{a}, \mathrm{b}, *}$, SAMAH F ABU-ZAID ${ }^{\mathrm{a}}$, SALAM A GHANAMEH ${ }^{\mathrm{a}}$ and N M GASANLY $^{\mathrm{c}}$ \\ ${ }^{a}$ Department of Physics, Arab-American University, Jenin, West Bank, Palestine \\ ${ }^{\mathrm{b}}$ Group of Physics, Faculty of Engineering, Atilim University, 06836 Ankara, Turkey \\ ${ }^{\mathrm{c}}$ Department of Physics, Middle East Technical University, 06800 Ankara, Turkey
}

MS received 13 February 2013; revised 20 March 2013

\begin{abstract}
The room temperature, dark and photo-dielectric properties of the novel crystals TIGaSeS are investigated in the frequency, intensity and biasing voltage having ranges of $\sim 1-120 \mathrm{MHz}, 14-40 \mathrm{klux}$ and 0-1 V, respectively. The crystals are observed to exhibit a dark high frequency effective dielectric constant value of $\sim 10.65 \times 10^{3}$ with a quality factor of $\sim 8.84 \times 10^{4}$ at $\sim 120 \mathrm{MHz}$. The dielectric spectra showed sharp resonance-antiresonance peaks in the frequency range of $\sim 25-250 \mathrm{kHz}$. When photoexcited, pronounced increase in the dielectric constant and in the quality factor values with increasing illumination intensity are observed. Signal amplification up to $\sim 33 \%$ with improved signal quality up to $\sim 29 \%$ is attainable via photoexcitation. On the other hand, the illuminated capacitance-voltage characteristics of the crystals reflected a downward shift in the voltage biasing and in the built-in voltage of the device that is associated with increase in the uncompensated carrier density. The increase in the dielectric constant with increasing illumination intensity is ascribed to the decrease in the crystal's resistance as a result of increased free carrier density. The light sensitivity of the crystals, the improved dielectric properties and the lower biasing voltage obtained via photoexcitation and the well-enhanced signal quality factor of the crystals make them promising candidates for optical communication systems.
\end{abstract}

Keywords. Varactor; TIGaSeS; sensors; dielectric.

\section{Introduction}

TlGaSeS crystals have attracted the interest of scientists for their remarkable electronic and optical properties. Optically, the crystals exhibited the coexistence of indirect and direct transitions with energy band gaps of $\sim 2 \cdot 27$ and $\sim 2.58 \mathrm{eV}$, respectively (Guler and Gasanly 2007). The rate of the change of the indirect band gap with temperature is reported to be $-3 \cdot 2 \times 10^{-4} \mathrm{eV} / \mathrm{K}$. On the other hand, the TlGaSeS crystals were previously reported to exhibit Schottky contacts. Two types of devices have been fabricated. The first design relates to two Ag contact points on the surface of the crystal (Qasrawi et al 2012) and the other relates to the Ag and carbon (Qasrawi and Gasanly 2012) contacts along the $c$-axis of the crystal. For the two Ag contacts and under low biasing voltage, the device exhibited a resonance-antiresonance phenomenon at a frequency of 27.5 and $34 \mathrm{kHz}$, respectively (Qasrawi et al 2012). The capacitance spectral analysis indicated discrete power storage, selectively of the device at particular frequencies. Such property of the device is promising for implantation in both radio and microwave technological applications. On the other hand, the $\mathrm{Ag}-\mathrm{C}$

\footnotetext{
*Author for correspondence (aqasrawi@atilim.edu.tr, aqasrawi@aauj.edu)
}

contacts along the $c$-axis of the crystal reflected a very high quality capacitance-frequency signal at $\sim 62.517 \mathrm{kHz}$. The energy loss for that device is much less than $0.001 \%$ of the stored value. This indicates the ability of using the device as power amplifier that in turn makes the TlGaSeS crystal promising material for technological application in the power sector (Qasrawi and Gasanly 2012).

$\mathrm{TlGaSeS}$ crystals are also reported to exhibit clearly resolved photoluminescence spectra with two emission bands at $\sim 1.605$ and $\sim 1.254 \mathrm{eV}$ (Gasanly 2011), the dielectric properties of this material is still not well investigated. Some articles about the composers $\left(\mathrm{TlGaSe}_{2}-\right.$ $\mathrm{TlGaS}_{2}$ ) of $\mathrm{TlGaSeS}$ crystals may be found (Sheleg et al 2003; Nair et al 2005). In addition to these works, an ellipsometrical analysis of the interband transitions in $\mathrm{TlGaS}_{(2 x)} \mathrm{Se}_{2(1-x)}$ has been reported in the frequency range of $2.9 \times 10^{14}-1.49 \times 10^{15} \mathrm{~Hz}$ (Isik and Gasanly 2012). The real and imaginary parts of the dielectric function, refractive index and extinction coefficient were calculated from ellipsometric data using the ambient-substrate optical model. Such a study is applicable at photonic frequencies and is helpful for optical responses. Here, in this work, we will investigate the dark and photoexcited dielectric properties of $\mathrm{TlGaSeS}$ crystals for the first time. The study aims to explain the variations in dielectric properties of this material as an important parameter for device fabrication. In addition, the attenuations in the 
dielectric loss which play vital role in electronic device applications, associated with the photoexcitation will be considered. Furthermore, the photo-biasing effect on the TlGaSeS as an electronic device will also be analysed.

\section{Experimental}

The crystal growth and electrical contact design were described in our previous works (Guler and Gasanly 2007; Qasrawi et al 2012). The dielectric constant of TlGaSeS crystal was recorded between two Ag contact points. The measurement was handled in the a.c. signal frequency range of $1 \mathrm{k}-120 \mathrm{MHz}$ under variable light intensities using an LCR meter and $0-120 \mathrm{MHz}$ function generator. The light was supplied from a halogen lamp. The light intensity was altered by varying position of the source.

\section{Results and discussion}

The dielectric constant of TlGaSeS crystals was studied as function of a.c. signal frequency in the dark and under different photoexcitation intensities. The room temperature data of effective dielectric constant $\left(\varepsilon_{\mathrm{eff}}\right)$ were registered in the frequency range of $1 \mathrm{k}-120 \mathrm{MHz}$. Figure 1 displays the variation of dielectric constant with applied a.c. signal frequency for a $0.23 \mathrm{~V}(0 \mathrm{dBm}$-the Bluetooth power) biasing voltage. For the dark and light excitation cases, the effective dielectric constant which is presented in the inset of figure 1(b) reflects some apparent sharp peaks at particular frequencies. The maximum peak (resonance peak) of the dark dielectric spectra appears at $\sim 32.6 \mathrm{kHz}$ and is followed by sharp minima (antiresonance) at $\sim 35 \mathrm{kHz}$. This physical phenomenon previously showed maxima at $\sim 27.5$ and minima at $\sim 34 \mathrm{kHz}$, when subjected to biasing voltage of $1.4 \mathrm{~V}$ (16 dBm-typical wireless LAN transmission power in laptops) (Qasrawi et al 2012). The shift in the spectra is simply due to the linear variation of spectral maximum frequency with applied voltage. Similarly, under photoexcitation, a clearly readable shift in the main resonance-antiresonance peak may be observed from the inset of figure 1(b).

The observed resonance-antiresonance phenomenon in the dark $\varepsilon_{\text {eff }}-f$ spectra was previously observed in the capacitance spectra (Qasrawi et al 2012) and was discussed and ascribed to the effective dielectric constant switching between the dynamic and static states of the device due to the limited unit cell size of the crystallites as being much less than the incident wavelength (Koschny et al 2003).

Figure 1( $a$ and $b)$ illustrate the real and imaginary parts of the dielectric constant in the dark and under various excitation intensities being presented in the frequency range of $1-100 \mathrm{kHz}$. As it is clearly observable from figure 1(a), the dark real dielectric constant gradually increases from $5 \cdot 17 \times 10^{3}$ at $\sim 1 \mathrm{kHz}$ to $10.92 \times 10^{3}$ at $\sim 29.7 \mathrm{kHz}$, where it sharply jumps to $14.09 \times 10^{3}$ at $\sim 32.6 \mathrm{kHz}$ (maximum resonance). Then, it decreases drastically to $7.76 \times 10^{3}$ at $\sim 35 \mathrm{kHz}$ (antiresonance) and re-increase reaching a constant value of $10.65 \times 10^{3}$ for all the studied frequency range $(53 \mathrm{k}-120 \mathrm{MHz})$. On the other hand, figure 1(b) displays imaginary part of the dielectric $\left(\varepsilon_{\mathrm{im}}\right)$ spectra being studied in the same frequency range. In contrast to the real part of the dielectric constant, the imaginary part continuously decreases with increasing applied signal frequency indicating the dielectric dispersion. However, as table 1 shows, because the imaginary part of dielectric constant is at least one order of magnitude less than the real part, its effect on the effective dielectric spectra is not pronounced. The dispersion in the dielectric constant may be explained by means of charge polarization and/or hopping models. In accordance to Maxwell-Wagner model (Wagner 1973), a dielectric medium is assumed to be composed of conducting crystallites (TlGaSeS layers) separated by resistive boundaries (between each two layers). When an a.c. signal is applied, charge carriers can easily migrate into the crystallites and accumulates at the crystallite boundaries. Such process causes large polarization and high dielectric constant values. The resistivity of the boundaries contributes to the high value of dielectric constant at low frequencies. In addition, the high value of the dielectric constant can also be explained on the basis of interfacial/ space charge polarization due to inhomogeneous dielectric structure. The inhomogeneity present near the crystal surface may exist due to broken bonds and layer mismatch (Kaur et al 2012).

Alternatively, when the charge transport takes place by hopping conduction, the motion of a charge is accompanied by an electrical relaxation. An ionic or electronic charge is surrounded by negative or positive counter charges. A hop to a new site can lead to a successful charge transport only if the polarization can follow the signal. Otherwise, the charge carrier will jump back. This mutual relaxation requires a relaxation time $(t)$. Thus, if the external electric field has a frequency, which is much higher than $(1 / t)$, it affects on the charge transport which then averages out. In contrast, when the applied signal frequency is much less than $(1 / t)$, it supports the charge transport and causes a contribution to the electric relaxation (Kremer and Schönhals 2003).

The inverse of the loss tangent (ratio of the imaginary to the real part of the dielectric constant) known as the quality factor, $Q$, is illustrated in figure 2. The dark $Q$ factor of the crystal continuously increases with increasing signal frequency. It reaches a value of $6.8 \times 10^{4}$ at $\sim 120 \mathrm{MHz}$. Such property indicates high energy storing ability.

Figure 1 also displays the effect of photoexcitation intensity on the effective, real and imaginary dielectric constant. A pronounced shift in the dielectric values 
Table 1. Dark and photoelectrical parameters of TlGaSeS crystals at $300 \mathrm{~K}$.

\begin{tabular}{lccccc}
\hline Intensity (klux) & $\varepsilon_{\mathrm{r}} \times 10^{3}$ & $\varepsilon_{\mathrm{im}} \times 10^{3}$ & $f_{\max }(\mathrm{kHz})$ & $V_{\mathrm{bi}}(\mathrm{V})$ & $N_{\mathrm{a}} \times 10^{12}\left(\mathrm{~cm}^{-3}\right)$ \\
\hline Dark & 14.09 & 0.551 & 32.6 & 0.472 & 5.26 \\
14 & 14.38 & 0.553 & 32.5 & & \\
19 & 14.96 & 0.590 & 32.4 & & \\
26 & 14.96 & 0.590 & 32.3 & 0.364 & 0.79 \\
31 & & & & 0.360 & 0.86 \\
36 & 16.39 & 0.632 & 31.8 & & 1.26 \\
38 & 16.97 & 0.632 & 31.6 & 0.352 & 1.71 \\
39 & 18.7 & 0.590 & 31.1 & & \\
40 & & &
\end{tabular}
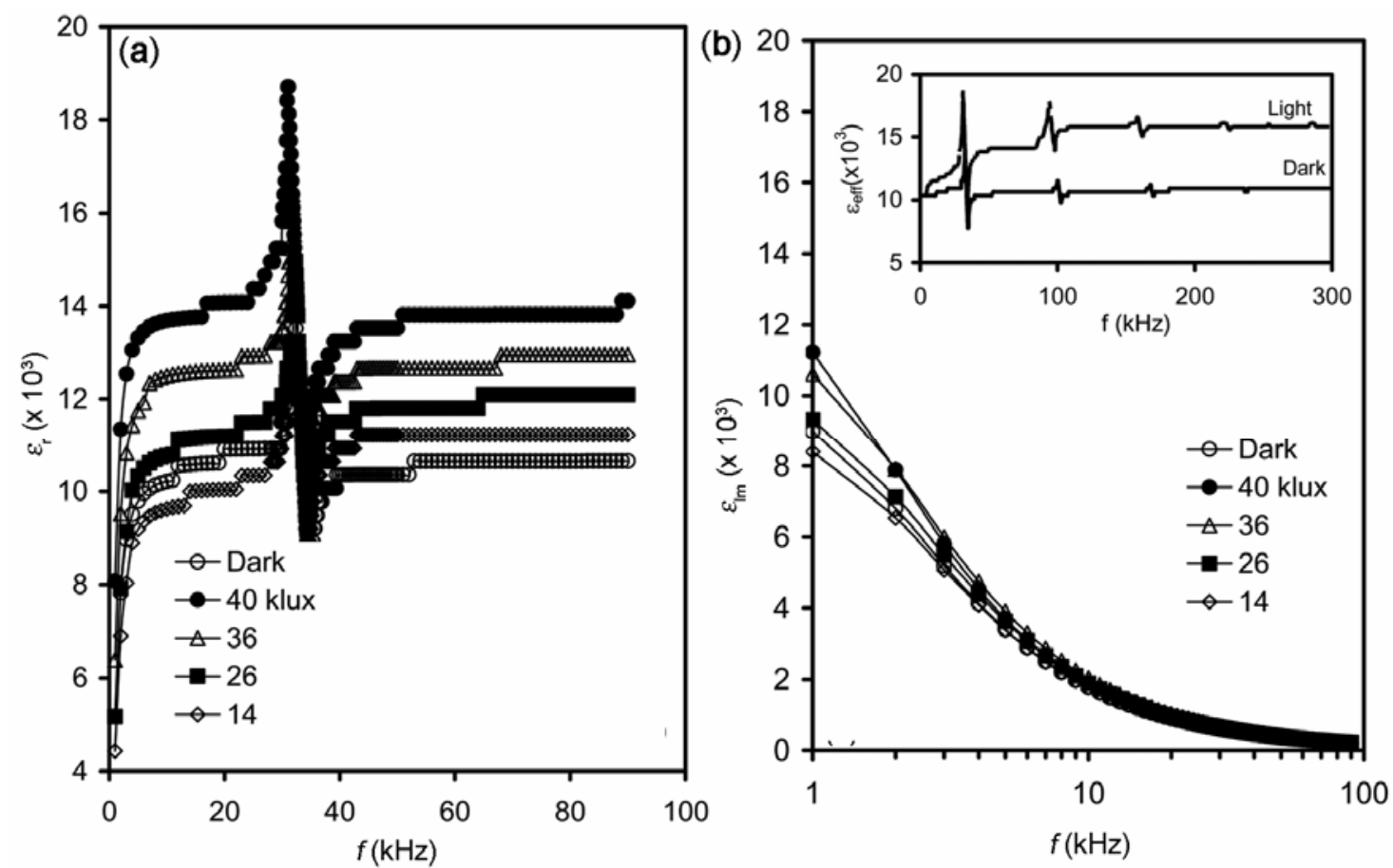

Figure 1. (a) Real dielectric constant spectra and (b) imaginary dielectric spectra at various illumination intensities for $\mathrm{TlGaSeS}$ crystals. Inset shows effective dielectric constant spectra.

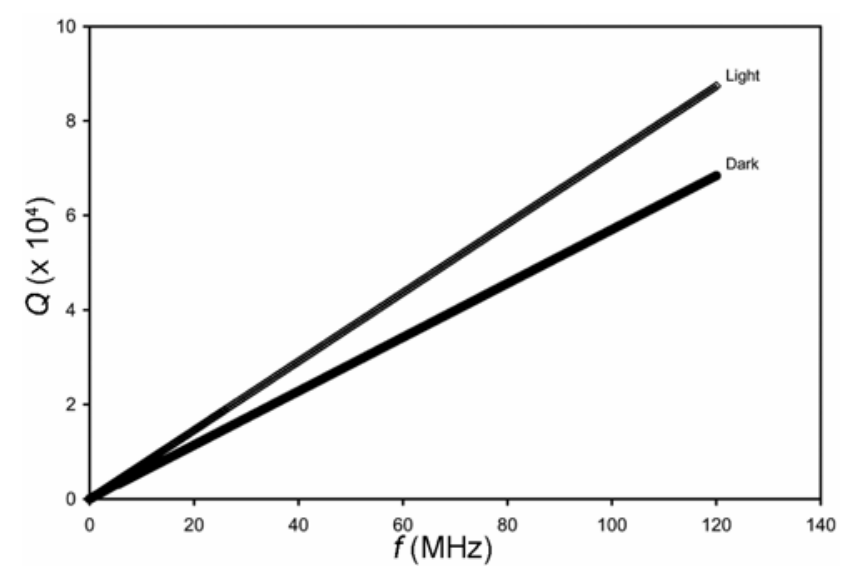

Figure 2. Dark and photoexcited quality factor and frequency dependence. toward lower frequencies is apparent from this figure. As table 1 displays, the real dielectric constant value increases from $14.09 \times 10^{3}$ in the dark to $16.39 \times 10^{3}$ at $\sim 36 \mathrm{klux}$ and it reaches $18.7 \times 10^{3}$ at $\sim 40$ klux. This increase in the dielectric constant is associated with a shift in the maximum peak frequency from $\sim 32.6$ in the dark to $\sim 31.1 \mathrm{kHz}$ under photoexcitation intensity of $\sim 40$ klux. The percentage increase from dark to $40 \mathrm{klux}$ in the real and imaginary dielectric constant values are 33 and $7 \%$, respectively. Consistently, it is clear from figure 2 that photoexcitation shifted the quality factor of the devices significantly. It exhibits a value of $\sim 8.8 \times 10^{4}$ at $\sim 120 \mathrm{MHz}$ and illumination intensity of 40 klux. The percentage increase in $Q$ factor is $\sim 29 \%$.

Figure 3(a) reflects the dark and photo-capacitancevoltage characteristics of the TlGaSeS crystals being 

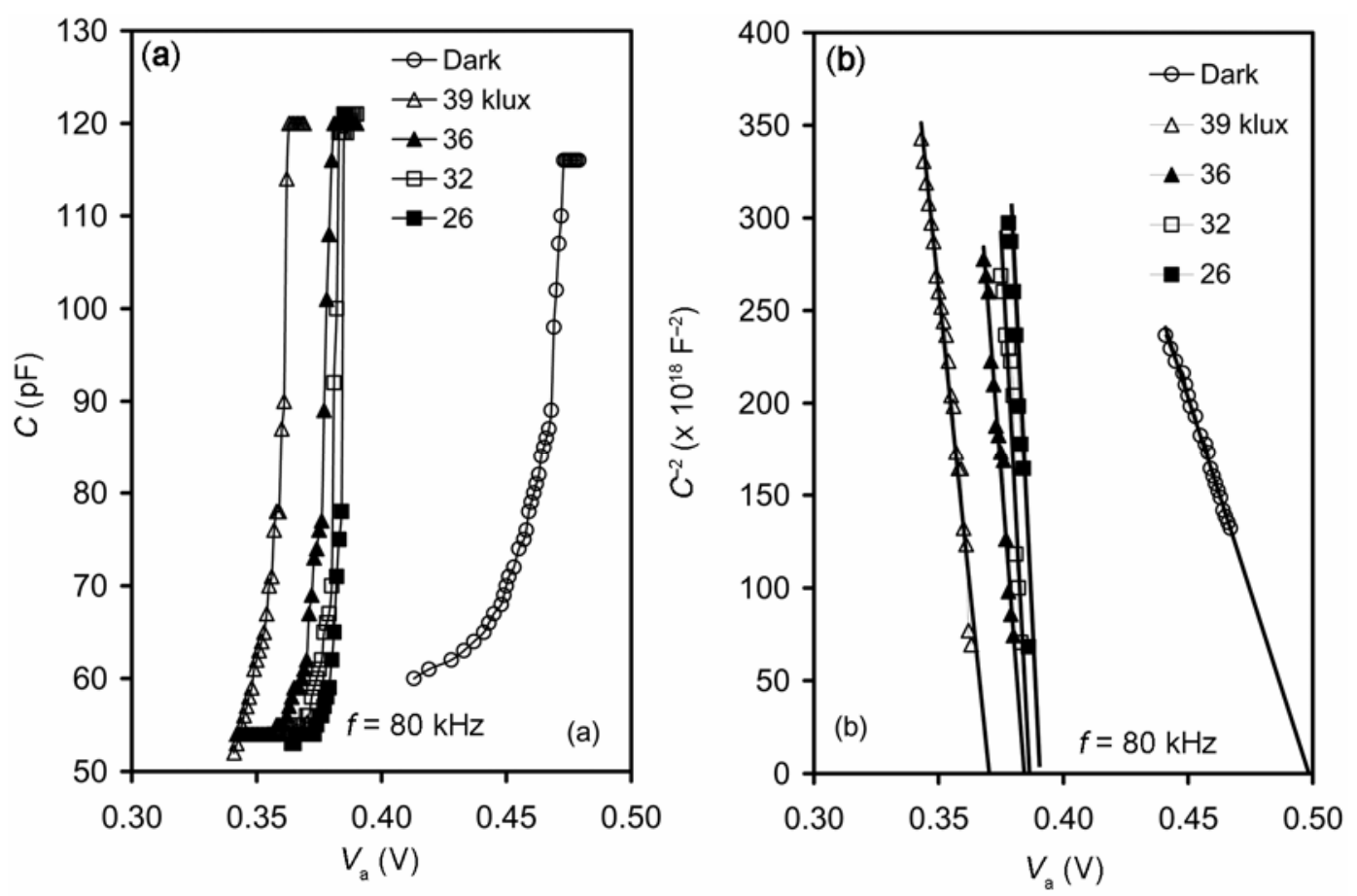

Figure 3. (a) $C-V$ characteristics at various illumination intensities and (b) plots of $C^{-2}-V_{\text {a }}$ dependence.

registered at $80 \mathrm{kHz}$ in the voltage range of $0-1 \mathrm{~V}$. For all curves and under applied voltages $\left(V_{\mathrm{a}}\right)$ less than $0 \cdot 34 \mathrm{~V}$ and greater than $0.48 \mathrm{~V}$, the capacitance is voltage invariant, which were not shown in the figure as they relate to the charge accumulation and inversion regions, respectively. The illustrated data refer to the depletion region of the device. One may read from the figure that the higher the light intensity, the more the shift toward lower applied voltage values in the $C-V$ characteristics. As shown in figure $3(\mathrm{~b})$, analysing the $C-V$ curves-in accordance to the depletion approximation-with the help of the MottSchottky model that leads (Tyagi 1991) to the calculation of the built-in voltage $\left(V_{\mathrm{bi}}\right)$ and the concentration of the noncompensated ionized carriers $\left(N_{\mathrm{a}}\right)$ through the equation

$$
C^{-2}=2\left(V_{\mathrm{bi}}+V_{\mathrm{a}}-k T / q\right) /\left(q \varepsilon_{\mathrm{s}} \varepsilon_{0} A^{2} N_{\mathrm{a}}\right),
$$

with $V_{\mathrm{a}}$ being the applied voltage, $\varepsilon_{\mathrm{s}}=7 \cdot 15$ is the static dielectric constant and $A=0.03 \mathrm{~cm}^{2}$, the device area. The plot of $1 / C^{2}$ vs $V_{\mathrm{a}}$ illustrated in figure 3(b) reveals the slopes and intercepts that allow determining the device parameters. The data are displayed in table 1. A systematic decrease in $V_{\mathrm{bi}}$ with increasing illumination intensity is observed. This shift is associated with increase in $N_{\mathrm{a}}$. Namely, for excitation intensity of 26 and 39 klux, the device exhibits a built-in voltage and carrier density of 0.364 and $0.344 \mathrm{~V}$ and $7.88 \times 10^{11}$ and $1.71 \times 10^{12} \mathrm{~cm}^{-3}$, respectively.

In an attempt to explain the changes in the dielectric parameters of the TlGaSeS crystals accompanied to the photoexcitation, we turn the attention to published works on $\mathrm{Al} / p$-GaAs/In barrier diodes (Soylu et al 2012) and InGaZnO thin film transistors (Hsieh et al 2012) as their results appear to be consistent with ours. Namely, for these materials, it is observed that the light illumination on the surface of the devices caused a shift in the capacitance spectra, the $C-V$ characteristic curves and in the loss tangent spectral curves (Hsieh et al 2012). Following the work of Goswami and Goswami (1973), Soylu et al (2012) has attributed the increase in the photocapacitance with illumination intensity to the increase in the carrier concentration. The increase of the charge carrier concentration under illumination causes an improvement in the space charge layer. The capacitance is described by the equation

$$
C=C_{\infty}+1 /\left((2 \pi f)^{2} R^{2} C_{\infty}\right),
$$

with $R$ being the resistance of the device and $C_{\infty}$ is the capacitance at the higher frequency. In accordance to this equation, the increase in capacitance, $C$ with illumination may be ascribed to the decrease in the value of the resistance arising from the increase in the free carrier concentration with illumination. On the other hand in another work, the increase in the capacitance with increasing light intensity was explained by means of dipole polarization mechanism (Dokme et al 2012). Particularly, in this mechanism, the dipolar polarization arising from permanent orientable dipoles, impurities and surface charges at intermediate frequencies (few $\mathrm{MHz}$ ) and interfacial polarization at low frequencies (few $\mathrm{kHz}$ ) is the main reason for the capacitance increase and built-in voltage 
shift. Usually, the interfacial polarization mechanism plays a role when the mobile charge carriers are impeded by a physical barrier that inhibits charge migration (Dokme et al 2012).

In another view, Demirezen et al (2013) assigned these behavioral changes to the generation of electron-hole pairs in the depletion region of the semiconductor. Physically, the generated electron (e)-hole (h) pair is stressed by electric field which causes $\mathrm{e}-\mathrm{h}$ pair separation. When it happens, electrons sweep out the semiconductor quickly by electric field, while holes are swept slowly allowing sufficiently long time for trapping process by incomplete bonds and defects. The process in turn leads to an additional photo-capacitance and conductance in the diode.

\section{Conclusions}

In this work, the dielectric properties of TlGaSeS single crystals are studied by means of capacitance spectra and capacitance voltage characteristics. In general, this material exhibits high dielectric constant values that increase upon light intensity increase. Due to its very low dielectric loss tangent (high quality factor), it is able to amplify the resonance signals up to remarkable percentages of $\sim 33 \%$. It was also observed that, the larger the light intensity, the lower the biasing voltage of the TlGaSeS device. The depletion region response to the applied voltage is relatively narrower indicating the fast response ability of the device. Such fascinating features of the crystal make its promising possible applications in electronics and optoelectronic devices.

\section{References}

Demirezen S, Altındal S and Uslu I 2013 Curr. Appl. Phys. 13 53

Dokme I, Yildiz D E and Altindal S E 2012 Adv. Polym. Technol. 3163

Gasanly N M 2011 J. Alloys Compd. $\mathbf{5 0 9} 4205$

Goswami A and Goswami A P 1973 Thin Solid Films 16 175

Guler I and Gasanly N M 2007 J. Kor. Phys. Soc. 512031

Hsieh T Y, Chang T C, Chen T C, Tsai M Y, Chen Y T, Chen Y, Chung, Ting H C and Chen C Y 2012 ECS J. Solid State Sci. Technol. 1 Q6

Isik M and Gasanly N M 2012 Opt. Commun. 2854092

Kaur J, Gupta V, Kotnala R K and Verma K C 2012 Ind. J. Pure Appl. Phys. 5057

Koschny T, Markoš P, Smith D R and Soukoulis C M 2003 Phys. Rev. E68 065602(R)

Kremer F and Schönhals A 2003 Broadband dielectric spectroscopy (Germany: Springer) p. 476

Nair K M, Guo R, Bhalla A S, Suvorov D and Hirano S I 2005 Advances in dielectric materials and electronic devices: proceedings of the 107th annual meeting of the American Ceramic Society (Balitmore, Maryland, USA)

Qasrawi A F, Elayyat S M S and Gasanly N M 2012 Cryst. Res. Technol. 47615

Qasrawi A F and Gasanly N M 2012 Mater. Sci. Eng. B177 981

Sheleg A U, Iodkovskaya K V and Kurilovich N F 2003 Phys. Solid State 4569

Soylu M, Al-Ghamdi A A and Yakuphanoglu F 2012 Microelectron. Eng. 9950

Tyagi M S 1991 Introduction to semiconductor materials and devices (New York: John Wiley \& Sons) p. 263

Wagner K W 1973 Am. J. Phys. 40317 